CERN-EP/2001-009

January 29. 2001

\title{
Silicon Detector for a Compton Camera in Nuclear Medical Imaging
}

\author{
D. Meier ${ }^{1}$, A. Czermak ${ }^{2}$, P. Jalocha ${ }^{2}$, B. Sowicki ${ }^{2}$, M. Kowal ${ }^{3}$, W. Dulinski ${ }^{4}$, G. Mæhlum ${ }^{5}$, E. Nygård $^{5}$, K. Yoshioka $^{5}$, J. Fuster $^{6}$, \\ C. Lacasta ${ }^{6}$, M. Mikuz ${ }^{7}$, S. Roe ${ }^{8}$, P. Weilhammer ${ }^{8}$, C-H. Hua ${ }^{9}$, S-J. Park ${ }^{9}$, S.J. Wildermann ${ }^{9}$, L. Zhang ${ }^{9}$, N.H. Clinthorne ${ }^{1}$, W.L. Rogers ${ }^{1}$ \\ 1 Medical School, University of Michigan, Ann Arbor, U.S.A. \\ 2 Niewodniczanski Institute of Nuclear Physics, Krakow, Poland \\ 3 Faculty of Physics and Nuclear Techniques, University of Mining and Metallurgy, Krakow, Poland \\ ${ }^{4}$ LEPSI, IN2P3/CNRS-ULP, Strasbourg, France \\ 5 Ideas ASA, Oslo, Norway \\ ${ }^{6}$ Inst. de Fisica Corpuscular, Universitat de Valencia, Valencia, Spain \\ 7 University of Ljubljana, Slovenia \\ 8 CERN, Geneva, Switzerland \\ 9 College of Engineering, University of Michigan, Ann Arbor, U.S.A.
}

Abstract - Electronically collimated gamma cameras based on Compton scattering in silicon pad sensors may improve imaging in nuclear medicine and bio-medical research. The work described here concentrates on the silicon pad detector developed for a prototype Compton camera. The silicon pad sensors are read out using low noise VLSI CMOS chips and novel fast triggering chips. Depending on the application a light weight and dense packaging of sensors and its readout electronics on a hybrid is required. We describe the silicon pad sensor and their readout with the newly designed hybrid. We also describe a modular and lowcost data acquisition system (CCDAQ) based on a digital signal processor which is interfaced to the EPP port of personal computers. Using the CCDAQ and the hybrids energy spectra of gamma-ray photons from technetium $\left({ }_{43}^{99 \mathrm{~m}} \mathrm{Tc}\right)$ and americium $\left({ }_{95}^{241} \mathrm{Am}\right)$ were acquired with an energy resolution of $2.45 \mathrm{keV}$ FWHM for the $140.5 \mathrm{keV}$ photo-absorption line of ${ }_{43}^{99 \mathrm{~m}}$ Tc. For all pads the discrimination threshold in the trigger chip was between (15 and 25) $\mathrm{keV}$.

Keywords - Compton camera, electronic collimation, silicon, sensor, low noise, self-triggering, charge sensitive, readout

\section{INTRODUCTION}

$\mathrm{C}$ OMPTON cameras have the potential to improve imaging of radio isotope labeled organs. The basic idea of a Compton camera is to replace mechanical collimators used regularly with clinical Anger cameras by electronic collimation using the Compton effect [1]. A Compton camera consists of two detectors: a Compton scatter detector and a position sensitive crystal scintillator. Photons emitted from the sample undergo Compton scattering with shell electrons inside the scatter detector where the position and kinetic energy, $T$, of the recoil electrons are measured. The scattered photons undergo photo-absorption inside the crystal scintillator where their position and total energy are measured. This article is dedicated to the development of the Compton scatter detector only, whereas descriptions about the development of the scintillator and image reconstruction can be found in references [2], [3] and [4].

\section{Compton Scatter Detector}

At present a prototype Compton scatter detector is being developed using silicon pad sensors with self triggering signal readout. Fig. 1 shows a photograph of the hybrid carrying a silicon pad sensor and its readout chips. In total eight pairs of VA/TA chips are mounted and wire bonded near the sides of the silicon sensor.

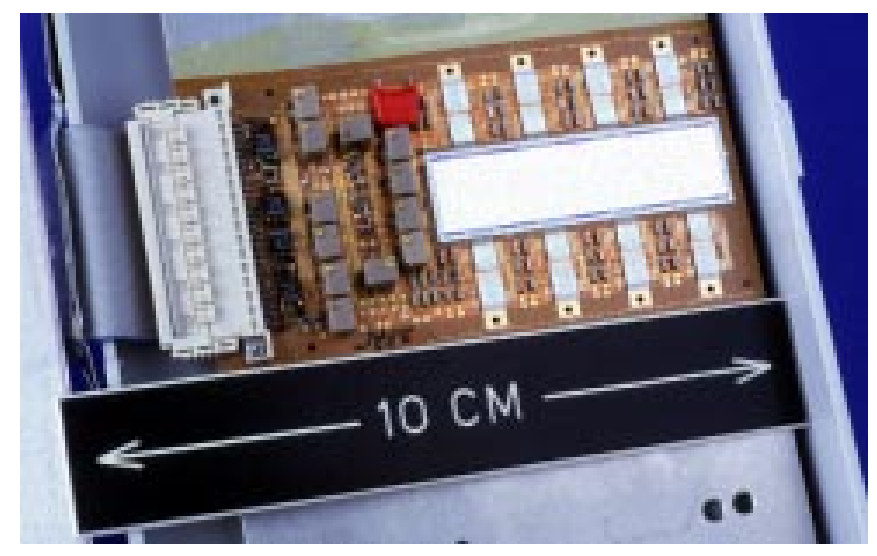

Fig. 1. Photograph of a hybrid carrying a silicon pad sensor and eight pairs of VA32C/TA32CG readout chips.

\section{A. Hybrid Preparation and Packaging}

The Compton scatter detector aims to maximize the amount of primary Compton interactions in the silicon and minimize the amount of secondary Compton interactions and photo-absorptions in the sensor itself and in support structure and electronics. Furthermore, future applications like Animal PET or Compton Probes require dense packaging of the silicon sensors inside the Compton scatter detector. Therefore hybrid boards have been manufactured using $50 \mu \mathrm{m}$ thin kapton foil with $150 \mu \mathrm{m}$ fine pitch copper lines on both sides. For the prototypes the kapton foil was glued onto an aluminum plate to ensure stability and enable cooling if required. The present design carries 
potentiometers and a large connector. In a future design these components as well as the readout electronics can be placed further away from the sensor to reduce the amount of mass in the vicinity of the silicon sensor.

\section{B. Silicon Pad Sensors}

The silicon pad sensors [5] were developed for the Compton scatter detector and a recent design can be seen in the center of the photograph in Fig. 1. At present $300 \mu \mathrm{m}$ thick silicon is used with an array of $8 \times 32=256$ pads at a pitch of $1.4 \times 1.4 \mathrm{~mm}^{2}$. Each pad is a $p^{+}$implant on high resistivity $(5 \mathrm{k} \Omega \mathrm{cm}) n^{-}$silicon with an area of $1.39 \times 1.39 \mathrm{~mm}^{2}$. The total sensitive area of the detector is $11.2 \mathrm{~mm} \times 44.8 \mathrm{~mm}$. The sensors were produced at CSEM [6]. Fig. 2 shows a schematic cross section of a silicon pad sensor. The pads of the metal-1 layer are AC coupled via a silicon-nitride and -oxide layer to the $p^{+}$padimplants. The pad-implants are punch-through biased via small $p^{+}$bias-implants. The bias-implants are connected to a common potential (normally at $0 \mathrm{~V}$ ). The pads of the metal-1 layer are connected to lines of the metal-2 layer which are routed to the front-end readout electronics at the sides of the sensor. Both metal-1 and metal-2 layer are separated by $\mathrm{a} \approx 5 \mu \mathrm{m}$ thick silicon oxide layer.

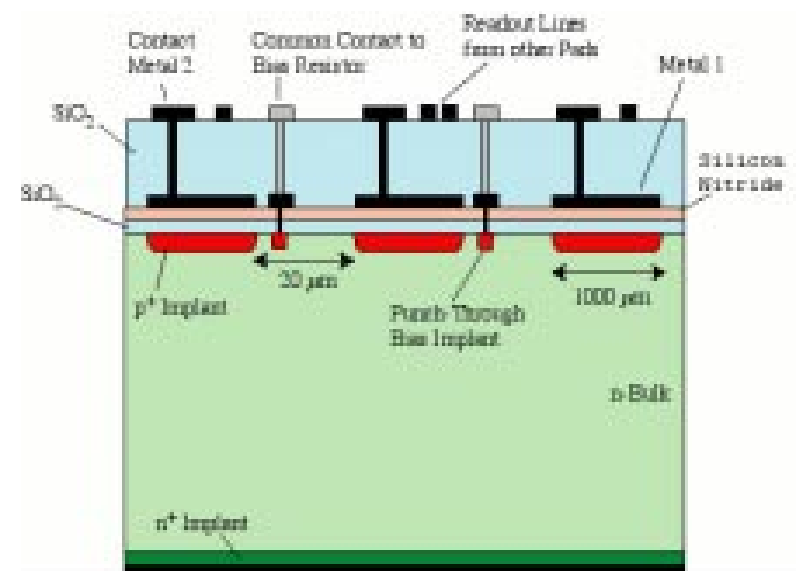

Fig. 2. Cross section view of a double-metal silicon pad sensor illustrating the implants and metal layers.

Fig. 3 shows a typical current-voltage curve of a silicon sensor before and after cutting from the 4" wafer. The positive bias voltage was applied to the backplane of the sensor. The bias current flows from the detector backplane through the bulk, via the punch-through resistors to the common contact, hence it is the total current through all 256 pads. The guard ring was at $0 \mathrm{~V}$ during the measurement and its current was recorded as well. It can be seen that the current over the punch-through bias resistors is $\approx 100 \mathrm{nA} / 256$ pads at $60 \mathrm{~V}$. The current over the guard ring is typically $\approx 25 \mathrm{nA}$. The behaviour before and after cutting is similar. Measurements indicate that the sensors are fully depleted for bias voltages greater than $55 \mathrm{~V}$. Several sensors were mounted and wire bonded to the readout electronics. Currents were $\approx 170 \mathrm{nA}$ at $55 \mathrm{~V}$ and at room temperature immediately after powering the readout chips. The current was observed to increase in time and to stabilize at $\approx 225 \mathrm{nA}$. At slightly higher environment temperature of $27^{\circ}$ the current stabilized typically around $280 \mathrm{nA}$. The increase in current is explained by an increase in temperature due to power dissipation from the readout chips. New wafers of similar design layout were recently delivered by Sintef [7]. First measurements on $0.5 \mathrm{~mm}$ thick sensors show a significantly reduced leakage current of $4 \mathrm{nA} / 256$ pads at a depletion voltage of $150 \mathrm{~V}$.

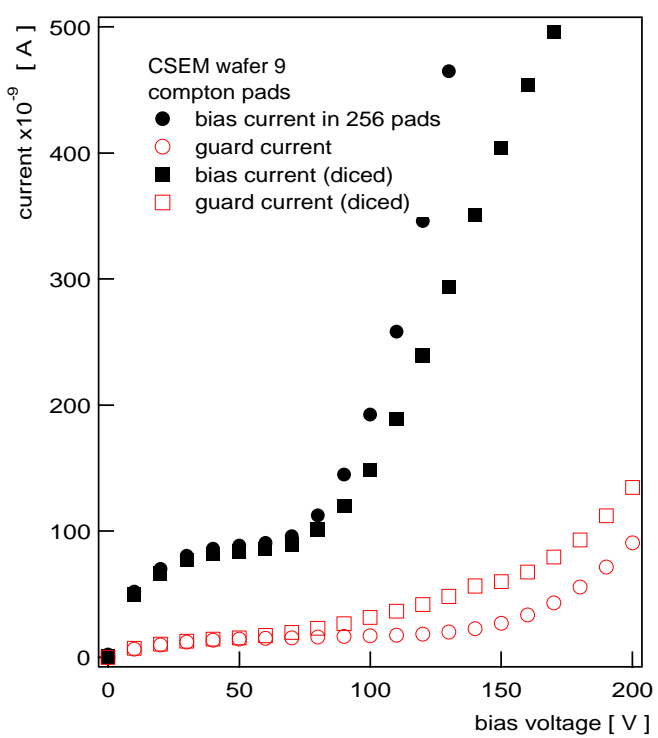

Fig. 3. Current-voltage characteristic in silicon pad sensors from CSEM measured at room temperature.

\section{VA/TA Signal Readout}

The Compton scatter detector requires a self triggering signal readout. For this task low noise charge sensitive amplifier chips (VA32C, a CMOS VLSI chip for 32 channel readout) and fast triggering chips (TA32CG) have been developed [8]. In the VA32C each channel contains a charge sensitive amplifier, a slow signal shaper $(2 \mu$ s peaking time) and a sample-and-hold followed by a multiplexer for all channels. In the TA32CG each channel obtains the signal from the charge sensitive amplifier of the VA32C, a shaper performs a 'fast' semi-Gaussian shaping (75 ns peaking time) which is followed by a signal discriminator. If the amplitude in one channel exceeds the discriminator threshold the readout of the $\mathrm{VA} 32 \mathrm{C}$ is triggered.

\section{C.1 Charge Sensitive Amplifier and Shaper (VA32C)}

Fig. 4 shows waveforms from a single channel of the VA32C (semi-Gaussian pulse shape) and the corresponding trigger signal from the TA32CG (rectangular pulse shape). The picture shows superimposed waveforms from signals caused by photons emitted by an americium gamma-ray source $\left({ }_{95}^{241} \mathrm{Am}\right)$. The spectral intensity is color coded and it can be seen that signals corresponding to the $59.5 \mathrm{keV}$ photo-absorption are most abundant. The shaping time of $2 \mu$ s can be read from the signal waveforms. 


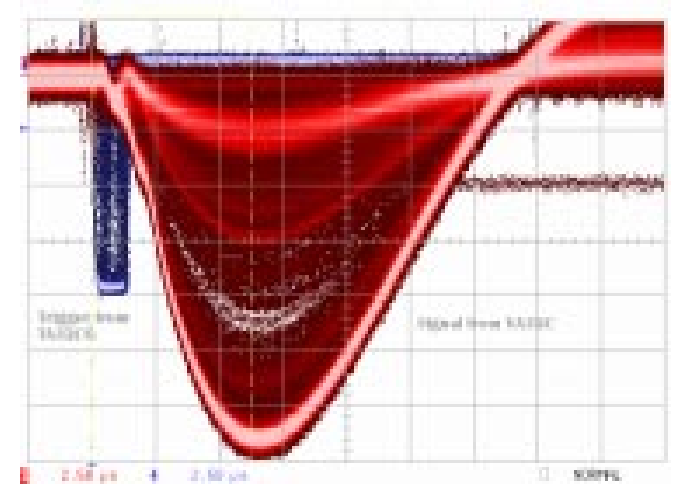

Fig. 4. Triggers from TA32CG (rectangular waveform) and signals from VA32C (semi-Gaussian waveform). The signals are from ${ }_{95}^{241} \mathrm{Am}$ with a photo-absorption line at $59.5 \mathrm{keV}$, visible on the scope in persistent display mode.

\section{C.2 Trigger Chip (TA32CG)}

The TA32CG is a successor of the TA32C. This new chip contains an adjustable gain stage. It also allows one to program a 3-bit DAC which controls the offset to the discriminator threshold individually for each channel - a feature used to align the trigger thresholds. The distribution of the trigger delay time between a charged particle interaction in the sensor and the TA32C trigger response was measured. For this measurement a plastic scintillator was mounted underneath the silicon sensor. A ${ }^{90} \mathrm{Sr}$ $\beta$-source was used to illuminate the sensor and the scintillator. Fig. 5 shows the distribution of the delay time between the signal from the scintillator and the trigger from one of the silicon pads. It can be seen that the TA32CG response time is nearly Gaussian distributed with a mean at about $100 \mathrm{~ns}$ (depending on the cable length) and a width of $17 \mathrm{~ns}$ FWHM. A narrow time distribution is desired in order to achieve a small coincidence time between the silicon and the second detector.

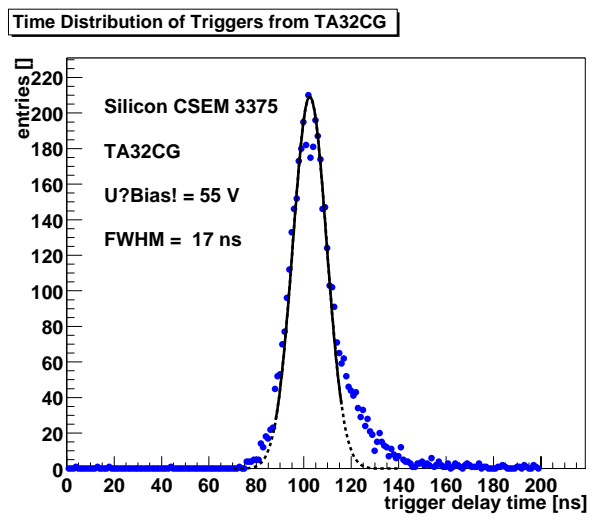

Fig. 5. Distribution of the trigger delay time.

\section{Results from Noise Measurements}

The angle reconstruction of the incoming photon requires the best possible angular resolution, $\Delta \theta$, depending in first order on the energy resolution, $\Delta T \approx \partial_{\theta} T(\theta) \Delta \theta$, and hence on the total electronic noise, $E N C_{\text {total }}$, in the readout: $\Delta T=2.35 \cdot 3.6 \mathrm{eV} / \mathrm{eh}-$ pair $\cdot E N C_{\text {total }}[e] \mathrm{FWHM}$. The noise on pads was measured to be on average $260 e$ at room temperature. From this noise one derives the energy resolution of $\Delta T=2.2 \mathrm{keV}$ FWHM. Measurements at temperatures below $+5^{\circ} \mathrm{C}$ reduce the sensor leakage current to $10 \mathrm{nA}$ and show an average noise of $160 e$ indicating that the detector leakage current contributes significantly to the noise. The corresponding energy resolution is $1.35 \mathrm{keV}$ FWHM. For $2 \mu$ s signal shaping the noise from the detector leakage current is $E N C_{\text {leakage }}[e] \approx 150 \times \sqrt{I_{\text {leakage }}[\mathrm{nA}]}$. Using the measured current of $\approx 225 \mathrm{nA} / 256$ pads one finds the equivalent noise of $141 \mathrm{e}$. The other important source for electronic noise in the readout is determined by series noise of the pre-amplifier depending on the total capacitance at its input. The electronic noise of the VA32C given by the manufacturer is at best $E N C_{\text {Amplifier }}=40 e+$ $12 e / \mathrm{pF}$ for $2 \mu$ s shaping time. The total capacitance seen by the charge sensitive amplifier was estimated to $4 \mathrm{pF} / \mathrm{pad}$ (including contributions from pad-to-backplane, pad-to-pad, metal-2 lines on top of the oxide and wirebonds). Hence one finds the equivalence noise charge of $E N C_{\text {Amplifier }} \approx 90 e$ which adds in quadrature to the leakage current noise giving an estimate on the total equivalent noise charge of $\approx 166 e$ at room temperature. The estimated value is still $94 e$ smaller than the measured value of $260 \mathrm{e}$. There could be sources for noise which are not yet considered in the estimation: a possible noise source is the series resistance of metal-2 lines, another possibility is that there are additional load capacitances. Both quantities are difficult to measure on the existing sensors.

\section{Data Acquisition System}

Several data acquisition units (CCDAQ-units) have been developed as prototypes for acquiring data from the silicon sensors. The goal was to acquire data from 256 pads with a speed of up to $5 \mathrm{kHz}$ in each unit. Fig. 6 shows a schematic view of the CCDAQ. Depending on the application there is a variable number of silicon sensors ( 1 to $\approx 10$ with 256 channels per sensor) foreseen to be read out by one CCDAQ-unit. The CCDAQ is connected to the EPP port of a personal computer. Up to 31 units can be attached to one EPP port.

Fig. 7 shows a photograph of one unit. Two printed circuit boards can be seen stacked above each other. The heart of the first board is a Motorola digital signal processors DSP56309. The DSP will be used to process and sparsify the raw data such that only hit position and energy are buffered and eventually transferred to the host computer. The second board contains the interface to the hybrid hosting a 12-bit bit analogue-to-digital converter and providing the power for the readout chips and various chip control voltages and currents. The power consumption of a single CCDAQ unit is $4.8 \mathrm{~W}$ and increases to about $6.5 \mathrm{~W}$ with one hybrid connected. The CCDAQ prototype units together with the new hybrids described above have been used to acquire first energy spectra from gamma-ray sources. 


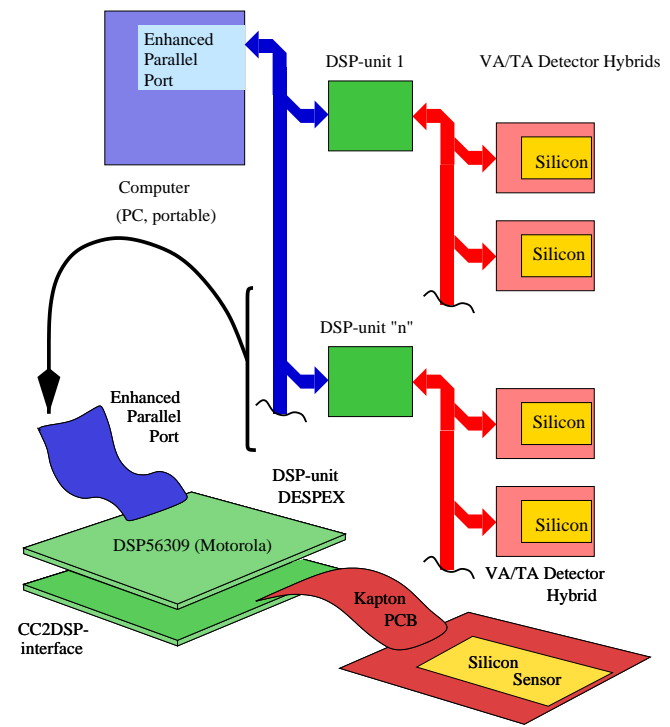

Fig. 6. Schematic view of the data acquisition, CCDAQ, for the Compton camera.

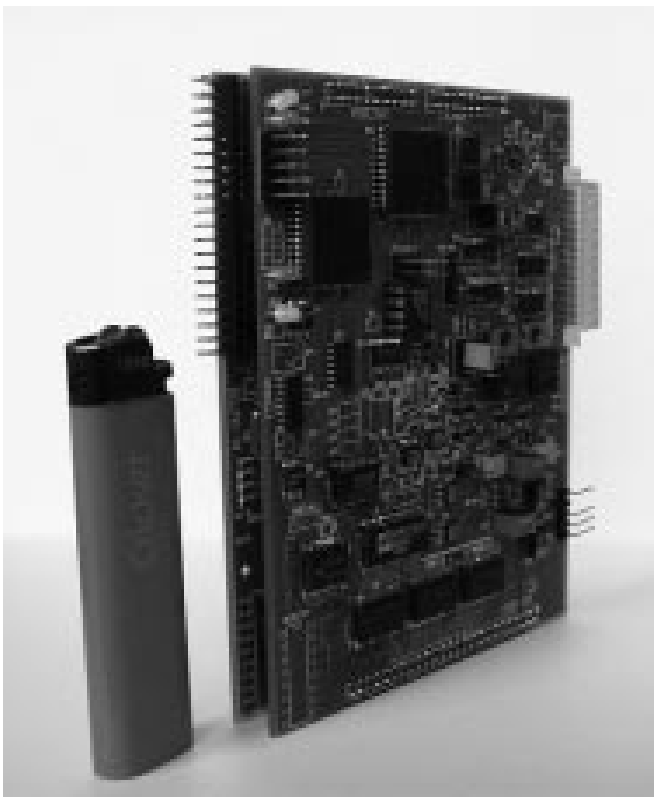

Fig. 7. Photograph of a data acquisition unit for the Compton camera.

\section{Results}

Energy spectra from a ${ }_{43}^{99 \mathrm{~m}} \mathrm{Tc}$ gamma source, commonly used in nuclear medicine, have been acquired at an environment temperature of $27^{\circ} \mathrm{C}$. The nucleus of ${ }_{43}^{99 \mathrm{~m}} \mathrm{Tc}$ emits gamma-ray photons with a most likely decay mode (in $88.9 \%$ of all cases) from a meta-stable state into its ground state releasing a photon with an energy of $140.5 \mathrm{keV}$. The meta-stable state has a half-life of 6.022 hours. There are also X-rays emitted with energies listed in Table I. A probe containing the Tc was mounted inside a lead collimator and was placed several centimeters above the silicon sensor, uniformly illuminating all pads. The discriminator thresholds were chosen to be as low as possible but sufficiently high to avoid triggering on electronic noise. If the signal amplitude in a pad exceeded its threshold the signal amplitudes of all 256 pads were sampled at their maximum, read out and digitized sequentially by the DSP. All raw data from pads were transferred to the host computer where further analysis (pedestal subtraction, common mode correction, and hit finding) was performed. After acquisition the channel-to-channel gain variations were corrected for each channel by normalizing the most significant photo-absorption line to the common mean value at 140.5 keV. Fig.8 (top) shows a two dimensional histogram where the ordinate gives the pad number and the abscissa gives the energy measured in the silicon sensor. The number of entries per bin is logarithmically grey-scale coded. The vertical line at $140.5 \mathrm{keV}$ illustrates the aligned gain over all 256 pads. Fig. 8 (bottom) shows the energy distribution from all pads using the same data from the top projected into a one dimensional energy histogram.
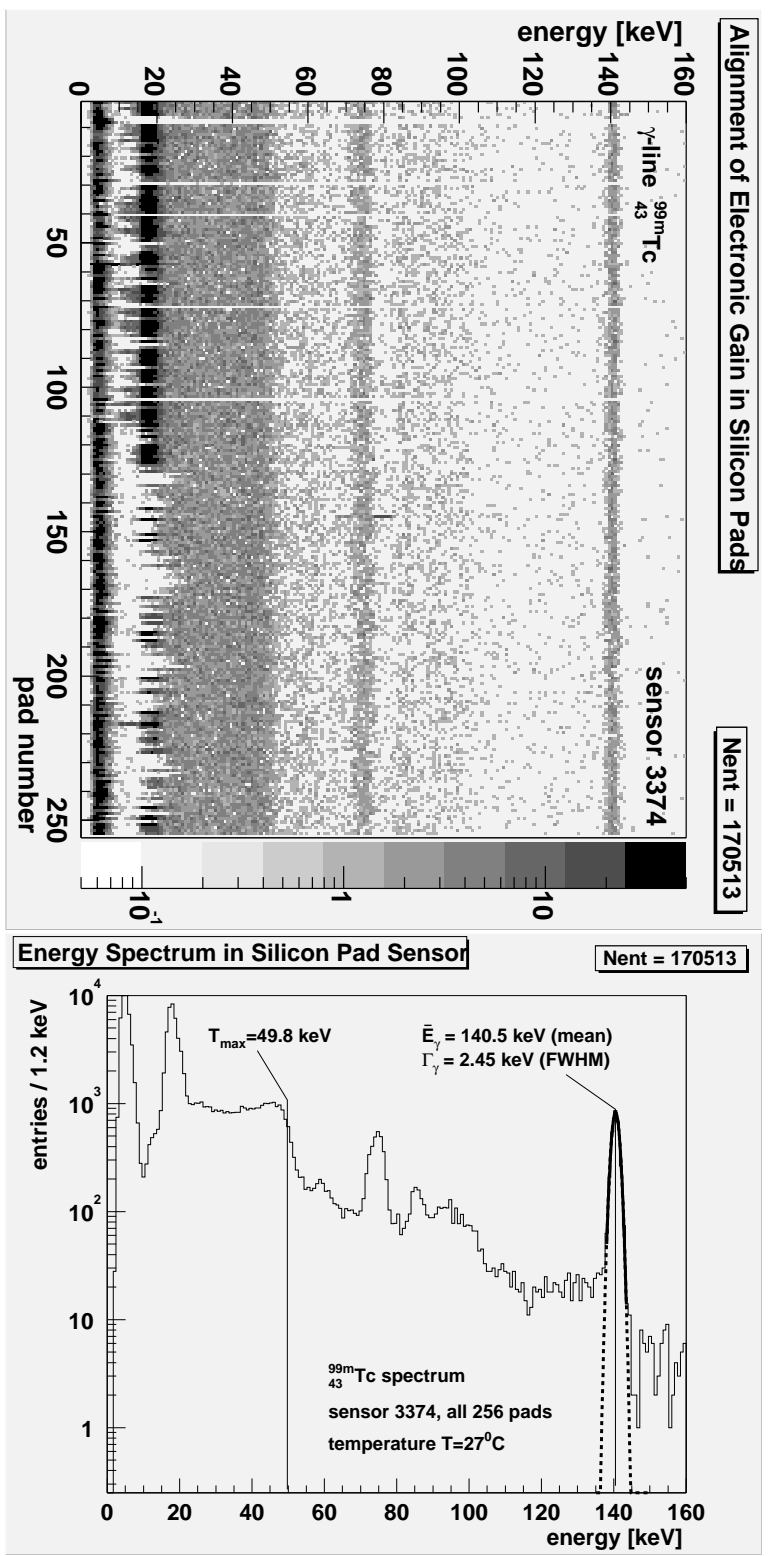

Fig. 8. Energy spectra from ${ }_{43}^{99 \mathrm{~m}} \mathrm{Tc}$ in silicon pads (top) and projection with spectra from all 256 pads included (bottom).

The most significant line with 4500 entries is visi- 
ble at $E_{\gamma}=140.5 \mathrm{keV}$ due to photo-absorption of the gamma-ray photons in the silicon. The distribution was fitted by a Gaussian function with $2.45 \mathrm{keV}$ FWHM energy resolution. From the measured electronic noise of $260 e$ (at room temperature) one derives an energy resolution of $2.2 \mathrm{keV}$ FWHM - slightly smaller than the result obtained in this spectrum. The difference is likely due to the higher environment temperature of $27^{\circ} \mathrm{C}$ during acquisition which may have caused a small increase in electronic noise. Around $18 \mathrm{keV}$ one can see events from $K_{\alpha 1, \alpha 2, \beta 1} \mathrm{X}$-rays (see Table I). The Compton continuum due to the $140.5 \mathrm{keV}$ gamma-rays is expected to start near zero energy and extend up to the Compton edge at $T_{\max }=2 E_{\gamma}^{2} /\left(m c^{2}+2 E_{\gamma}\right)=49.8 \mathrm{keV}$. The Compton continuum is clearly visible in the measured spectrum with a cut-off at the low end due to the discriminator thresholds and smeared out at the high end due to electronic noise and the Doppler effect. There are several lines near $75 \mathrm{keV}$ and $85 \mathrm{keV}$ which are caused by lead X-rays from the collimator. The measured energies for these lines are in agreement with values found in the literature as listed in Table I. We note that the threshold starts at $15 \mathrm{keV}$ for the first 128 channels of the detector and only starts at about $20 \mathrm{keV}$ for the other 128 channels. The threshold on the second half of the detector can improve by adjusting the programmable offsets for the threshold in the TA32CG chip.

\section{TABLE I}

$\mathrm{X}$-ray and gamma-ray energies from literature and the calculated Compton edge, $T_{\max }$, needed to explain the measured spectrum in Fig. 8.

\begin{tabular}{|l|c|c|c|c|c|}
\hline \multirow{2}{*}{ element } & \multicolumn{3}{|c|}{ X-ray energy $[\mathrm{keV}]$} & gamma & $T_{\max }$ \\
& $K_{\alpha 1}$ & $K_{\alpha 2}$ & $K_{\beta 1}$ & ray $[\mathrm{keV}]$ & {$[\mathrm{keV}]$} \\
\hline${ }_{43}^{99 \mathrm{~m}} \mathrm{Tc}$ & 18.367 & 18.251 & 20.619 & 140.5 & 49.8 \\
\hline${ }_{82}^{207} \mathrm{~Pb}$ & 74.969 & 72.804 & 84.936 & - & - \\
\hline
\end{tabular}

Fig. 9 shows the energy resolution on pads. The measurement has been done using gamma-rays from an ${ }_{95}^{241} \mathrm{Am}$ source. The resolution was obtained from a Gaussian fit to the photo-absorption line at $59.5 \mathrm{keV}$ where the upper plot also shows the errors from the fit to the data in each channel. The lower figure shows the energy resolution greyscale coded in the range from $1.8 \mathrm{keV}$ to $2.8 \mathrm{keV}$ versus the position in the sensor. It can be seen that the energy resolution varies among pads from values as low as $2.0 \mathrm{keV}$ up to $2.9 \mathrm{keV}$ with an average energy resolution of $2.4 \mathrm{keV}$.

\section{Summary}

We presented the status of a Compton scatter detector using silicon pad sensors with self triggering readout of low noise amplifier chips. First spectra have been acquired using a light weight hybrid board made of kapton. Kapton has been chosen in order to reduce mass in the Compton scatter detector. New trigger chips (TA32CG) have been
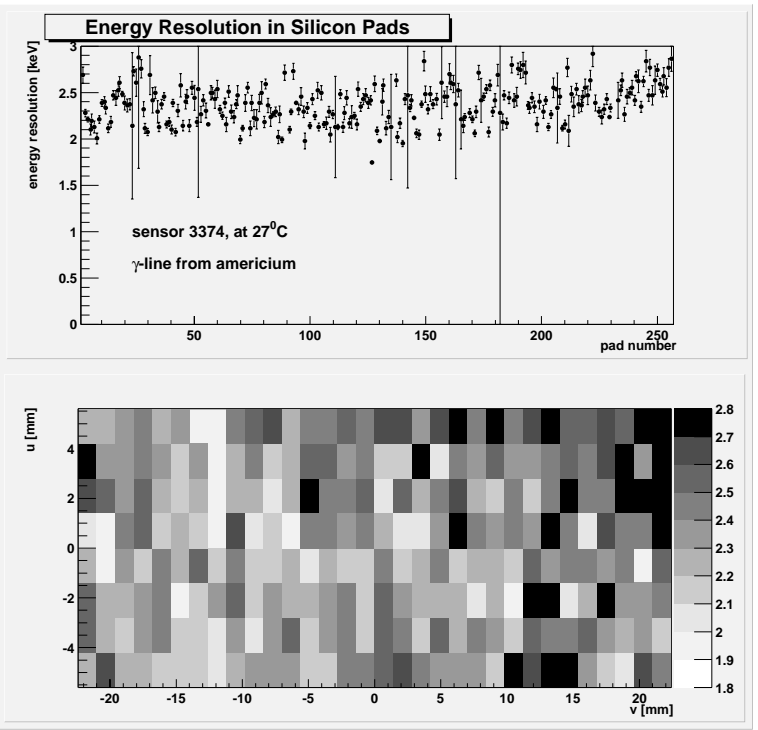

Fig. 9. Energy resolution versus pad number (top) and versus position in the sensor (bottom). The resolution was measured by a fit to the photo-absorption peak at $59.5 \mathrm{keV}$ from ${ }_{95}^{241} \mathrm{Am}$.

used with adjustable thresholds via a programmable DAC in each channel which allows one to align thresholds and effectively lower the overall threshold. The first measurements used thresholds in the range from (15 to 25$) \mathrm{keV}$. Using more refined methods the alignment is expected to improve in the future. An average energy resolution of $2.45 \mathrm{keV}$ FWHM was measured for the $140.5 \mathrm{keV}$ photoabsorption line from ${ }_{43}^{99 \mathrm{~m}} \mathrm{Tc}$. For the sensors used in this work the energy resolution was dominated by the sensor leakage current. The goal is an energy resolution near $1 \mathrm{keV}$ FWHM which can be approached using sensors with much lower leakage current. Such sensors were delivered by Sintef and results will be available in the near future. A new data acquisition system has been developed and prototypes have been used to acquire the first spectra shown here. This configuration of hybrids and acquisition system will be developed further in order to acquire images in the existing configuration of C-SPRINT or for new projects like Animal PET and Compton Probes.

\section{ACKNOWLEDGEMEnts}

At CERN we would like to thank A. Honma and K. Mühlemann for accurate wire bonding and A. Gandi and R. De Oliveira for their excellent work in kapton- and printed circuit board production.

\section{REFERENCES}

[1] M. Singh et al. "An Electronically Collimated Gamma Camera for Single Photon Emission Computed Tomography". Medical Physics, 10 (1983) 4.

[2] W. Rogers et al. "SPRINT II, A Second Generation Single Photon Ring Tomograph". IEEE, Trans. Med. Imaging, 7 (1988) 4.

[3] J.W. LeBlanc. "C-SPRINT: A Prototype Compton Camera System for low Energy Gamma Ray Imaging". IEEE, Trans. Nucl. Sci., 45 (1998) 3.

[4] S.J. Wilderman. "Fast Algorithm for List Mode Back-Projection of Compton Camera Data". IEEE, Trans. Nucl. Sci., 45 (1998) 3. 
[5] P. Weilhammer et al. "Si Pad Detectors". Nucl. Instr. Meth., 383 (1996) 89-97.

[6] CSEM. Centre Suisse d'Electronique et de Microtechnique SA. Rue Jaquet-Droz 1, P.O. Box 41, CH-2007 Neuchtel, Switzerland.

[7] SINTEF. Foundation of Scientific and Industrial Research at the Norwegian Institute of Technology, Electronics and Cybernetics, Microsystems. http://www.oslo.sintef.no/ecy/. Oslo and Trondheim, Norway.

[8] Integrated Detector and Electronics (Ideas ASA) http://www.ideas.no. Pb.315, Veritasveien 9, N-1322 Høvik, Norway, Tel: $++47-6755-1818$. 